\title{
THE PERMANENTAL PROCESS
}

\author{
PETER MCCULLAGH, ${ }^{*}$ University of Chicago \\ JESPER MøLLER, ${ }^{* *}$ Aalborg University
}

\begin{abstract}
We extend the boson process first to a large class of Cox processes and second to an even larger class of infinitely divisible point processes. Density and moment results are studied in detail. These results are obtained in closed form as weighted permanents, so the extension is called a permanental process. Temporal extensions and a particularly tractable case of the permanental process are also studied. Extensions of the fermion process along similar lines, leading to so-called determinantal processes, are discussed.

Keywords: Boson process; Cox process; density of spatial point process; determinantal process; factorial moment measure; fermion process; Gaussian process; infinite divisibility; simulation; spatio-temporal process; weighted permanent
\end{abstract}

2000 Mathematics Subject Classification: Primary 60G55

Secondary $62 \mathrm{M} 30$

\section{Introduction}

Cox process models for spatial point processes form a rich class of models for aggregated point patterns (see, e.g. [5], [6], [9], [24], and [29]). In applications a spatial point pattern is observed within a bounded window $S \subset \mathbb{R}^{d}$, and a Cox process restricted to $S$ is a finite random subset $\boldsymbol{X}$ of $S$ whose distribution is usually specified indirectly using a nonnegative random intensity function $\boldsymbol{\Lambda} \equiv(\Lambda(x))_{x \in S}$ such that $\int_{S} \Lambda(x) \mathrm{d} x<\infty$ almost surely. Given the random intensity function, $\boldsymbol{X}$ is a Poisson process on $S$ with intensity $\boldsymbol{\Lambda}$, so the marginal density of the point process $\boldsymbol{X}$ is

$$
f(\boldsymbol{x})=\mathrm{E}\left[\exp \left(|S|-\int_{S} \Lambda(x) \mathrm{d} x\right) \prod_{j=1}^{n} \Lambda\left(x_{j}\right)\right]
$$

for finite point configurations $\boldsymbol{x}=\left\{x_{1}, \ldots, x_{n}\right\} \subset S$ (see, e.g. [24]). Here $n(\boldsymbol{x}) \equiv n$, the number of points in $\boldsymbol{x}$, can be any nonnegative integer; the points in $\boldsymbol{x}$ are pairwise different; the density is with respect to the unit-rate Poisson process on $S$; the expectation is with respect to $\Lambda$; and $|S|$ is the volume of $S$.

For most models so far considered in the literature, apart from simple cases such as that of a mixed Poisson process where $\boldsymbol{\Lambda}$ is the product of a positive random variable and a nonnegative deterministic function, the expectation in (1) is computable only by Markov chain Monte Carlo methods. Examples are shot noise Cox processes [3], [23] and log Gaussian Cox processes

Received 2 September 2005; revision received 17 September 2006.

* Postal address: Department of Statistics, University of Chicago, 5734 University Avenue, Chicago, IL 60637, USA.

Email address: pmcc@galton.uchicago.edu

** Postal address: Department of Mathematical Sciences, Aalborg University, Fredrik Bajers Vej 7G, DK-9220 Aalborg,

Denmark. Email address: jm@math.auc.dk 
[25]. Indeed, closed-form expressions for densities of other kinds of spatial point process model are also unknown, apart from trivial cases closely related to Poisson processes. For example, for the important class of Markov point processes [31], the normalizing constant of the density cannot be evaluated explicitly.

Boson and fermion processes [2], [17], [18] and their extensions [27] are the major exception, in that analytic expressions are available for the density and the moments. In the first version of this paper, [21], we were not aware of the long and technical paper [27], which in many ways covers the results of the present paper. Since our approach is more natural and more direct, it may have more appeal to statisticians and probabilists. The present paper gives a unified exposition, which includes the proofs of the results, points to the results of [27], and highlights new facts.

The main difference between [27] and our paper is that the authors of the former began with a certain determinantal expansion due to Vere-Jones [32] and showed that it is the Laplace transform of a point process. We work in the reverse direction, beginning with an explicit Cox process which extends the boson process, and obtaining its moments, deriving its density function, and extending it to a class of infinitely divisible point processes called permanental processes.

\subsection{The permanental process as a Cox process}

The absence of a closed form for the density (1) motivated us to study a class of flexible Cox process models with intensity functions defined, for any positive integer $k$ and real covariance function $C\left(x, x^{\prime}\right), x, x^{\prime} \in S$, by

$$
\Lambda(x)=Z_{1}(x)^{2}+\cdots+Z_{k}(x)^{2},
$$

where $\boldsymbol{Z}_{j} \equiv\left(Z_{j}(x)\right)_{x \in S}, j=1, \ldots, k$, are independent, zero-mean Gaussian processes with covariance function $C / 2$ (as in [27], we assume for simplicity that the covariance matrix is real; most statements are similar for complex Gaussian processes). From the viewpoint of applications, this point process is similar to the log Gaussian Cox process. The main mathematical difference is that $\boldsymbol{\Lambda}$ has a convergent moment generating function whereas the $\log$ Gaussian process does not. As a result, the density function for the Cox process $\boldsymbol{X}$ is available analytically in the form of a weighted matrix permanent. Consequently, $\boldsymbol{X}$ is called a permanental process with parameters $\alpha=k / 2$ and $C$.

The boson (or photon) process [6], [11], [17], [18] corresponds to the $\alpha=1$ case. Another special case is that of a mixed Poisson process, i.e. $C\left(x, x^{\prime}\right)=c$ is constant and, hence, $\Lambda(x) \sim(c / 2) \chi^{2}(k)$ does not depend on $x \in S$.

The moments and the density of the Cox process were obtained in [27] from the expansion of a certain Fredholm determinant, which is in fact the moment generating function of $\boldsymbol{\Lambda}$. In the arguments that follow, the moments, cumulants, generating functions, and densities are derived directly.

\subsection{Permanents}

For any points $x_{1}, \ldots, x_{n} \in S$, the symbol $[C]\left(x_{1}, \ldots, x_{n}\right)$ denotes the $n \times n$ matrix with $(i, j)$ th entry $C\left(x_{i}, x_{j}\right)$. The key building block is the sum of cyclic products

$$
\operatorname{cyp}[C]\left(x_{1}, \ldots, x_{n}\right)=\sum_{\{\sigma: \# \sigma=1\}} C\left(x_{1}, x_{\sigma(1)}\right) \cdots C\left(x_{n}, x_{\sigma(n)}\right),
$$


where $\sigma$ is a permutation of $\{1, \ldots, n\}$ and $\# \sigma$ is the number of cycles. For real $\alpha$, the $\alpha$-weighted permanent ( $\alpha$-permanent)

$$
\operatorname{per}_{\alpha}[C]\left(x_{1}, \ldots, x_{n}\right)=\sum_{\sigma} \alpha^{\# \sigma} C\left(x_{1}, x_{\sigma(1)}\right) \cdots C\left(x_{n}, x_{\sigma(n)}\right)
$$

is the sum over all permutations (see [32]). This is a polynomial in $\alpha$ of degree $n$ in which the coefficient of degree $r$ is the sum of products over permutations having exactly $r$ cycles. The usual permanent has $\alpha=1$ (see [22]).

To each permutation $\sigma$ of $\{1, \ldots, n\}$ there corresponds a partition $B$ of the index set $\{1, \ldots, n\}$, each cycle of $\sigma$ generating a block of $B$. Thus, the $\alpha$-permanent can be expressed, in the form of a sum over the partition lattice $\mathcal{B}_{n}$, as

$$
\operatorname{per}_{\alpha}[C]\left(x_{1}, \ldots, x_{n}\right)=\sum_{B \in B_{n}} \alpha^{\# B} \prod_{b \in B} \operatorname{cyp}[C]\left(\boldsymbol{x}^{(b)}\right),
$$

where \#B is the number of blocks, and $\boldsymbol{x}^{(b)}$ is the set of $x$-values in block $b \in B$. Consequently, as will be demonstrated later, the relationship between cyclic products and the $\alpha$-permanent is precisely the relationship between cumulants and moments.

Valiant [30] has shown that the exact computation of permanents of general matrices is a sharp-P-complete problem, so no deterministic polynomial-time algorithm is available. However, polynomial-time algorithms exist for certain special cases, such as that of general fixedrank matrices [1], and for approximate Monte Carlo computation of general nonnegative matrices [16]. For most statistical purposes, approximate computation of permanent ratios, which is a less demanding task, is sufficient. In addition, analytic approximations are available for large $\alpha$. Statistical aspects and algorithms for calculating weighted permanents, permanent ratios, and likelihoods will be discussed in more detail in future work.

\subsection{Outline}

The product moments and the density of the permanental process are established in Section 2 under the assumption that $C$ is continuous and $2 \alpha$ is a positive integer. Section 3 shows that if certain weak conditions are satisfied (e.g. $C$ need not be a covariance function), a point process $X$ with a density of the same form exists for each $\alpha \geq 0$ and is infinitely divisible. This process is a Poisson randomization and there exists a nontrivial conditional limit process as $\alpha \rightarrow 0$, given that $\boldsymbol{X}$ is not empty. The Poisson randomization provides an easy way of simulating the process. Section 4 discusses temporal extensions of the permanental process. The special permanental process in which $C$ is proportional to a projection is discussed in Section 5; it is deemed special on account of its striking and unusual properties. Section 6 concludes the paper with a discussion on how to extend the fermion process along similar lines as was the boson process, whereby so-called determinantal point processes [14], [28] are obtained. While the permanental process is attractive, the determinantal process is repulsive.

\section{The permanental Cox process}

Throughout Sections 2.1-2.3, $2 \alpha$ is assumed to be a positive integer, so the permanental process $\boldsymbol{X}$ is the Cox process driven by (2).

\subsection{Moment properties}

For a finite point process with density $f$ with respect to the unit-rate Poisson process $\Phi$ on $S$, the $n$ th-order product density (see, e.g. [18] and [29, p. 111]) is given by $\rho^{(n)}\left(x_{1}, \ldots, x_{n}\right)=$ 
E $f\left(\Phi \cup\left\{x_{1}, \ldots, x_{n}\right\}\right)$ for any $n \in \mathbb{N}$ and pairwise-different points $x_{1}, \ldots, x_{n} \in S$. Intuitively, $\rho^{(n)}\left(x_{1}, \ldots, x_{n}\right) \mathrm{d} x_{1} \cdots \mathrm{d} x_{n}$ is the probability of observing $n$ points from $\boldsymbol{X}$ occurring jointly in each of $n$ infinitesimally small balls with centres $x_{1}, \ldots, x_{n}$ and volumes $\mathrm{d} x_{1}, \ldots, \mathrm{d} x_{n}$. Moreover, the $n$ th-order factorial moment measure has density $\rho^{(n)}$ with respect to the Lebesgue measure on $\mathbb{R}^{d n}$.

Since $\boldsymbol{X}$ is a Cox process driven by (2), its $n$ th-order product density is given by

$$
\rho^{(n)}\left(x_{1}, \ldots, x_{n}\right)=\mathrm{E}\left[\Lambda\left(x_{1}\right) \cdots \Lambda\left(x_{n}\right)\right] .
$$

Theorem 1, below, shows that this is a weighted permanent.

\subsubsection{General results}

Lemma 1. Let $(Z(x))_{x \in S}$ be a zero-mean, real Gaussian process with covariance function $C / 2$. For any not necessarily distinct $x_{1}, \ldots, x_{n} \in S$, the joint cumulant of order $n$ of the variables $Z\left(x_{1}\right)^{2}, \ldots, Z\left(x_{n}\right)^{2}$ is

$$
\operatorname{cum}_{n}\left(Z\left(x_{1}\right)^{2}, \ldots, Z\left(x_{n}\right)^{2}\right)=\operatorname{cyp}[C]\left(x_{1}, \ldots, x_{n}\right) / 2 .
$$

Proof. This is a standard application of the lattice-sum formula [19], [20] for generalized cumulants as a sum of products of ordinary cumulants. All Gaussian cumulants are 0 except those of order two, so the result is a sum of products of covariances, $C_{i_{1}, j_{1}} \cdots C_{i_{n}}, j_{n}$, where $C_{i, j} \equiv C\left(x_{i}, x_{j}\right)$. Since each value $1, \ldots, n$ occurs once as a first index and once as a second index, $\left(j_{1}, \ldots, j_{n}\right)$ is a permutation of $\left(i_{1}, \ldots, i_{n}\right)$. Noncyclic permutations do not satisfy the lattice connectivity condition, so the sum is restricted to cyclic permutations. For each cyclic permutation, there are $2^{n-1}$ distinct partitions of the $2 n$ indices that satisfy the connectivity condition, all giving rise to the same product, $C_{1, \sigma(1)} \cdots C_{n, \sigma(n)} / 2^{n}$. Consequently, the joint cumulant is one-half the sum of the cyclic products.

Theorem 1. For any not necessarily distinct $x_{1}, \ldots, x_{n} \in S$,

$$
\mathrm{E}\left[\Lambda\left(x_{1}\right) \cdots \Lambda\left(x_{n}\right)\right]=\operatorname{per}_{\alpha}[C]\left(x_{1}, \ldots, x_{n}\right) .
$$

Proof. Since $\Lambda(x)$ in (2) is the sum of $k$ independent, identically distributed (i.i.d.) random processes, the joint cumulant of order $n$ of $\Lambda\left(x_{1}\right), \ldots, \Lambda\left(x_{n}\right)$ is $k$ times the joint cumulant of $Z\left(x_{1}\right)^{2}, \ldots, Z\left(x_{n}\right)^{2}$, which is given by (3). The joint moment of order $n$ is the sum of cumulant products over subpartitions of $\{1, \ldots, n\}$, one cumulant for each block of the partition. Since permutation cycles determine the blocks of the partition, the number of blocks is equal to the number of cycles. As a result, the term corresponding to the permutation $\sigma$ has a factor $(k / 2)^{\# \sigma}$, so the sum is the weighted permanent with weight $\alpha=k / 2$.

In the complex version of Theorem $1, Z_{1}, \ldots, Z_{k}$ are independent, zero-mean, complex Gaussian processes with covariance function

$$
\operatorname{cov}\left(Z_{r}(x), \bar{Z}_{s}\left(x^{\prime}\right)\right)=\delta_{r s} C\left(x, x^{\prime}\right),
$$

where $C$ is Hermitian. The joint cumulant of $\left|Z_{1}\left(x_{1}\right)\right|^{2}, \ldots,\left|Z_{1}\left(x_{n}\right)\right|^{2}$ in Lemma 1 is thus $\operatorname{cyp}[C]\left(x_{1}, \ldots, x_{n}\right)$, and the joint moment in Theorem 1 is $\operatorname{per}_{k}[C]\left(x_{1}, \ldots, x_{n}\right)$. The result for $\alpha=\frac{1}{2}$ was given in [15], the result for $\alpha=1$ was given in [17] and [18], and the result in the case where $2 \alpha$ is a positive integer was given in [27, Theorem 4.1]. 
2.1.2. Generating functions. Let $(Z(x))_{x \in S}$ be a zero-mean, real Gaussian process with covariance function $C / 2$. For any not necessarily distinct $x_{1}, \ldots, x_{n} \in S$, the joint moment generating function of the variables $Z\left(x_{1}\right)^{2}, \ldots, Z\left(x_{n}\right)^{2}$ is

$$
M(t)=\operatorname{Eexp}\left(t_{1} Z\left(x_{1}\right)^{2}+\cdots+t_{n} Z\left(x_{n}\right)^{2}\right)=\left|I-t[C]\left(x_{1}, \ldots, x_{n}\right)\right|^{-1 / 2},
$$

where $t=\operatorname{diag}\left\{t_{1}, \ldots, t_{n}\right\}$ is a diagonal matrix and $I$ denotes the identity matrix.

Since $\boldsymbol{\Lambda}$ in (2) is a sum of $k=2 \alpha$ independent processes, the joint cumulant generating function of $\Lambda\left(x_{1}\right), \ldots, \Lambda\left(x_{n}\right)$ is

$$
\log M(t)=-\alpha \log \left|I-t[C]\left(x_{1}, \ldots, x_{n}\right)\right| .
$$

Since this function has a Taylor expansion about the origin, it follows from the calculations in Section 2.1.1 that the cumulant coefficients in the Taylor expansion are cyclic products.

As it happens, $M(t)$ is also proportional to the probability generating function for certain multivariate binomial and negative binomial distributions (see [13]). The coefficients in the expansion of this determinant were obtained in [32] in the form of $\alpha$-permanents. The connection with Gaussian processes was noted in [27]. With a suitably modified covariance function, $M(t)$ is both the Laplace transform and the probability generating function of the associated Cox process. A more general operator expansion of the Fredholm determinant, clarifying these relationships and showing that the point process has product densities in the form of $\alpha$-permanents, was given in [27].

\subsection{Conditions}

In Section 2.3, and also sometimes later on, we assume that $S$ is compact and that the restriction of the covariance function $C$ to $S \times S$ is a real, continuous function. Thus, by Mercer's theorem (see, e.g. [8, p. 344]), the covariance function has spectral representation

$$
C\left(x, x^{\prime}\right)=\sum_{r=0}^{\infty} \lambda_{r} e_{r}(x) e_{r}\left(x^{\prime}\right), \quad x, x^{\prime} \in S,
$$

with absolute and uniform convergence in $S \times S$; the eigenvalues satisfy $\lambda_{0} \geq \lambda_{1} \geq \cdots \geq 0$ and

$$
\operatorname{tr}(C):=\int_{S} C(x, x) \mathrm{d} x=\sum_{r=0}^{\infty} \lambda_{r}<\infty
$$

(i.e. the trace of $C$ is finite); the eigenfunctions $e_{r}$ form an orthonormal basis of $\mathcal{L}_{2}(S)$, the space of square-integrable, real Borel functions on $S$ with inner product $\langle p, q\rangle=\int_{S} p(x) q(x) \mathrm{d} x$; and $e_{r}$ is continuous if $\lambda_{r} \neq 0$. We take $Z_{j}(x)=\sum_{r=0}^{\infty} V_{j, r} e_{r}(x)$, where the $V_{j, r}$ are independent $\mathrm{N}\left(0, \lambda_{r} / 2\right)$-distributed random variables. Clearly $Z_{1}, \ldots, Z_{k}$ are then independent, zero-mean Gaussian processes with covariance function $C / 2$. By (2), (7), and Fubini's theorem,

$$
\mathrm{E} n(\boldsymbol{X})=\mathrm{E} \int_{S} \Lambda(x) \mathrm{d} x=\alpha \operatorname{tr}(C)
$$

is finite, and

$$
\int_{S} \Lambda(x) \mathrm{d} x=\sum_{j=1}^{k} \sum_{r=0}^{\infty} \sum_{s=0}^{\infty} V_{j, r} V_{j, s}\left\langle e_{r}, e_{s}\right\rangle=\sum_{j=1}^{k} \sum_{r=0}^{\infty} V_{j, r}^{2}
$$

is almost surely finite. 
The rank of $C$ is the number of nonzero eigenvalues. Note that $C$ is a projection if and only if each $\lambda_{r}$ is either 1 or 0 , and (7) implies that then $\operatorname{rank}(C)<\infty$. For $C$ proportional to a projection of rank one, the permanental process is a mixed Poisson process.

For functions $h: S^{n} \rightarrow[0, \infty)$, we define

$$
\int_{S^{n}} h(\boldsymbol{x}) \mathrm{d} \boldsymbol{x}=\int_{S} \cdots \int_{S} h\left(x_{1}, \ldots, x_{n}\right) \mathrm{d} x_{1} \cdots \mathrm{d} x_{n},
$$

where for $n=0$ we interpret the integral as $h(\varnothing), \varnothing$ being the empty point configuration. Furthermore, if $h\left(x_{1}, \ldots, x_{n}\right)$ is a symmetric function, we do not distinguish between $\boldsymbol{x}$ as a vector and $\boldsymbol{x}$ as a point configuration. Indeed product densities, weighted permanents, and many other functions considered in the sequel are symmetric functions, and it is often convenient to write $\rho^{(n)}(\boldsymbol{x}), \operatorname{per}_{\alpha}[C](\boldsymbol{x}), \operatorname{cyp}[C](\boldsymbol{x})$ and so on for a finite point configuration $\boldsymbol{x} \subset S$ of $n(\boldsymbol{x})=n$ points. Finally, we set

$$
\rho^{(0)}(\varnothing)=\operatorname{per}_{\alpha}[C](\varnothing)=1, \quad \operatorname{cyp}[C](\varnothing)=0 .
$$

\subsection{Density function}

Let the situation be as in Section 2.2. To derive the density of the permanental process, it is convenient to introduce

$$
\tilde{\lambda}_{r}=\lambda_{r} /\left(1+\lambda_{r}\right), \quad r=0,1, \ldots,
$$

and

$$
\tilde{C}\left(x, x^{\prime}\right)=\sum_{r=0}^{\infty} \tilde{\lambda}_{r} e_{r}(x) e_{r}\left(x^{\prime}\right), \quad x, x^{\prime} \in S,
$$

which, by combining the proof of Mercer's theorem, the properties of $C$ (see Section 2.2), and the fact that $0 \leq \tilde{\lambda}_{r} \leq \lambda_{r}$, we can see to be a well-defined and continuous covariance function. Furthermore, we define $\tilde{\boldsymbol{\Lambda}}=(\tilde{\Lambda}(x))_{x \in S}, \tilde{\boldsymbol{X}}$, and $\tilde{\rho}^{(n)}$ in the same way as $\boldsymbol{\Lambda}=(\Lambda(x))_{x \in S}, \boldsymbol{X}$, and $\rho^{(n)}$, respectively, except that we replace $C$ by $\tilde{C}$. Finally,

$$
D:=\sum_{r=0}^{\infty} \log \left(1+\lambda_{r}\right)=-\sum_{r=0}^{\infty} \log \left(1-\tilde{\lambda}_{r}\right)
$$

is well defined, since $0 \leq \log \left(1+\lambda_{r}\right) \leq \lambda_{r}$ and $\sum \lambda_{r}<\infty$.

Theorem 2. The density of the permanental process $\boldsymbol{X}$ at any finite point configuration $\boldsymbol{x} \subset S$ is

$$
f(\boldsymbol{x})=\mathrm{e}^{|S|-\alpha D} \operatorname{per}_{\alpha}[\tilde{\boldsymbol{C}}](\boldsymbol{x}) .
$$

Proof. Combining (1) and (8) yields

$$
f(\boldsymbol{x})=\mathrm{e}^{|S|} \mathrm{E}\left[\left(\prod_{j=1}^{k} \prod_{r=0}^{\infty} \mathrm{e}^{-V_{j, r}^{2}}\right)\left(\prod_{j=1}^{n} \Lambda\left(x_{j}\right)\right)\right],
$$

where (recall) $V_{j, r} \sim \mathrm{N}\left(0, \lambda_{r} / 2\right), j=1, \ldots, k, r=0,1, \ldots$, are independent random variables. If $g_{r}$ is the density of $\mathrm{N}\left(0, \lambda_{r} / 2\right)$ and $\tilde{g}_{r}$ is the density of $\mathrm{N}\left(0, \tilde{\lambda}_{r} / 2\right)$, then $\mathrm{e}^{-v^{2}} g_{r}(v)=$ $\tilde{g}_{r}(v) /\left(1+\lambda_{r}\right)^{1 / 2}$. Consequently,

$$
\mathrm{E}\left[\left(\prod_{j=1}^{k} \prod_{r=0}^{\infty} \mathrm{e}^{-V_{j, r}^{2}}\right)\left(\prod_{j=1}^{n} \Lambda\left(x_{j}\right)\right)\right]=\mathrm{E}\left[\prod_{j=1}^{n} \tilde{\Lambda}\left(x_{j}\right)\right] \prod_{r=0}^{\infty}\left(1+\lambda_{r}\right)^{-\alpha} .
$$

Hence, from (4), we obtain (10). 
Theorem 2 was first established for the special case of the boson process $(\alpha=1)$ by Macchi [17], [18], who noted that $\tilde{C}$ is the root of the integral equation

$$
\tilde{C}\left(x, x^{\prime}\right)+\int_{S} \tilde{C}(x, y) C\left(y, x^{\prime}\right) \mathrm{d} y=C\left(x, x^{\prime}\right) .
$$

For the case where $2 \alpha$ is a positive integer, see [27, Theorem 6.17].

\subsection{Convolution and superposition}

Let $h$ be a symmetric function defined on finite subsets $x \subset S$ and, for each real $\alpha$ and nonempty configuration $\boldsymbol{x}=\left\{x_{1}, \ldots, x_{n}\right\}$, let

$$
H_{\alpha}(\boldsymbol{x})=\sum_{B \in B_{n}} \alpha^{\# B} \prod_{b \in B} h\left(\boldsymbol{x}^{(b)}\right) .
$$

Here, $\boldsymbol{x}^{(b)} \subset \boldsymbol{x}$ is the subset corresponding to block $b \in B$. The convention for the empty set is $h(\varnothing)=0$ and $H_{\alpha}(\varnothing)=1$. Formally, $H_{\alpha}(\boldsymbol{x})$ is the moment determined by the cumulants $\alpha h(\boldsymbol{w}), \boldsymbol{w} \subseteq \boldsymbol{x}$. Functions of this type are important in the theory of point processes because they satisfy the convolution property

$$
\begin{aligned}
\sum_{\boldsymbol{w} \subseteq \boldsymbol{x}} H_{\alpha}(\boldsymbol{w}) H_{\alpha^{\prime}}(\overline{\boldsymbol{w}}) & =\sum_{B \in \mathcal{B}_{w}} \sum_{B^{\prime} \in \mathcal{B}_{\bar{w}}} \alpha^{\# B}\left(\alpha^{\prime}\right)^{\# B^{\prime}} \prod_{b \in B} \prod_{b^{\prime} \in B^{\prime}} h\left(\boldsymbol{x}^{(b)}\right) h\left(\boldsymbol{x}^{\left(b^{\prime}\right)}\right) \\
& =\sum_{B \in \mathcal{B}_{\boldsymbol{x}}} \prod_{b \in B} h\left(\boldsymbol{x}^{(b)}\right) \sum_{A \subseteq B} \alpha^{\# A}\left(\alpha^{\prime}\right)^{\# \bar{A}} \\
& =\sum_{B \in \mathcal{B}_{\boldsymbol{x}}}\left(\alpha+\alpha^{\prime}\right)^{\# B} \prod_{b \in B} h\left(\boldsymbol{x}^{(b)}\right) \\
& =H_{\alpha+\alpha^{\prime}}(\boldsymbol{x}),
\end{aligned}
$$

where $\mathcal{B}_{\boldsymbol{x}}$ is the set of partitions of $\boldsymbol{x}$ and $\overline{\boldsymbol{w}}$ is the complement of $\boldsymbol{w}$ in $\boldsymbol{x}$. Note that, in the second line of the above display, $A \subseteq B$ and $\bar{A} \subseteq B$ are complementary subsets of $B$, so \#A is the number of blocks of $B$ that occur in $A$. Either subset, $A$ or $\bar{A}$, could be empty.

One consequence of the convolution property is that if $H_{\alpha}$ is the product density of a point process $\boldsymbol{X}$ and $H_{\alpha^{\prime}}$ is the product density of an independent process $\boldsymbol{X}^{\prime}$, then $H_{\alpha+\alpha^{\prime}}$ is the product density of the superposition process $\boldsymbol{X} \cup \boldsymbol{X}^{\prime}$. In addition, for any $\theta>0$, the function $\theta^{\alpha} H_{\alpha}(\boldsymbol{x})$ also satisfies the convolution property, and it is in this form that the result applies to the convolution of densities in (10). We shall exploit (11) later.

\subsection{Positivity conjectures}

If $2 \alpha$ is a positive integer, $\operatorname{per}_{\alpha}[C](\boldsymbol{x})$ is the expected value of a positive random variable and is thus positive for every finite point configuration $x \subset S$. Let $g(C)$ be the set of real $\alpha$ for which $\operatorname{per}_{\alpha}[C](\boldsymbol{x}) \geq 0$ at all point configurations $\boldsymbol{x} \subset S$. Evidently $g(C)$ is an additive semigroup containing the nonnegative half-integers. If $C\left(x, x^{\prime}\right)$ is nonnegative then $g(C)$ contains all $\alpha \geq 0$ and the natural questions are, is $\operatorname{per}_{\alpha}[C](\boldsymbol{x})$ an unnormalized density with respect to the unit-rate Poisson process? and, if so, what is the normalizing constant?

In fact, for each $\alpha, 0<\alpha<\frac{1}{2}$, it is usually possible to find a symmetric, positive, semidefinite matrix $A=[C](\boldsymbol{x})$ such that $\operatorname{per}_{\alpha} A<0$, and [27, Conjecture 7.2] states that this is always possible. We have been unable to prove [27, Conjecture 7.1], namely that $\operatorname{per}_{\alpha}[C](\boldsymbol{x})$ is nonnegative for noninteger values of $2 \alpha \geq 1$. However, extensive numerical work points to the 
following conjecture regarding the roots of the permanent polynomial for nonzero, symmetric, positive, semidefinite matrices $A$ : for complex numbers $\alpha$,

$$
\operatorname{per}_{\alpha}(A)=0 \quad \Longrightarrow \quad \operatorname{Re}(\alpha)<\frac{1}{2} .
$$

\section{Extensions}

\subsection{Conditions}

In the remainder of the paper, unless otherwise stated we relax the conditions in Section 2.2 and define $C$ in terms of $\tilde{C}$ as follows.

Denote by $\mathbb{R}_{+}$the set of nonnegative real numbers. We assume that $\alpha \in \mathbb{R}_{+}, S$ is compact, and $\tilde{C}: S^{2} \rightarrow \mathbb{R}_{+}$is symmetric and continuous. Here $\tilde{C} \geq 0$ ensures that $\operatorname{per}_{\alpha}[\tilde{C}] \geq 0$ for all $\alpha \in \mathbb{R}_{+}$, which is needed in Theorem 3, below. By the spectral theorem, for Lebesgue-almost all $\left(x, x^{\prime}\right) \in S \times S$,

$$
\tilde{C}\left(x, x^{\prime}\right)=\sum_{r=0}^{\infty} \tilde{\lambda}_{r} e_{r}(x) e_{r}\left(x^{\prime}\right),
$$

where the eigenfunctions $e_{r}$ form an orthonormal basis of $\mathcal{L}_{2}(S), e_{r}$ is continuous if $\tilde{\lambda}_{r} \neq 0$, and $\left|\tilde{\lambda}_{0}\right| \geq\left|\tilde{\lambda}_{1}\right| \geq \cdots$. Note that the eigenvalues $\tilde{\lambda}_{r}$ are now allowed to be negative (a simple example is given in Section 3.3). We also assume that the spectral norm $\|\tilde{C}\|=\left|\tilde{\lambda}_{0}\right|$ is strictly less than 1 , and that $\sum_{r=0}^{\infty}\left|\tilde{\lambda}_{r}\right|$ is finite. These assumptions are of course satisfied when $\tilde{C}$ is a continuous covariance function (cf. Section 2.2). The assumptions imply absolute and uniform convergence of the series in (12) for Lebesgue-almost all $\left(x, x^{\prime}\right) \in S \times S$.

For integers $r \geq 2$, recursively define the continuous and symmetric function

$$
\tilde{C}^{r}\left(x, x^{\prime}\right)=\int_{S} \tilde{C}^{r-1}\left(x, x^{\prime \prime}\right) \tilde{C}\left(x^{\prime \prime}, x^{\prime}\right) \mathrm{d} x^{\prime \prime}=\sum_{j=0}^{\infty} \tilde{\lambda}_{j}^{r} e_{j}(x) e_{j}\left(x^{\prime}\right),
$$

where $C^{0} \equiv 1$ and the second equality follows from the spectral theorem and the continuity of $\tilde{C}$. Note that, for integers $r \geq 2$,

$$
\tilde{C}^{r}\left(x, x^{\prime}\right) \leq\|\tilde{C}\|^{r-2} \sum_{j=0}^{\infty} \tilde{\lambda}_{j}^{2}\left|e_{j}(x)\right|\left|e_{j}\left(x^{\prime}\right)\right|,
$$

where by Mercer's theorem the sum is a finite constant, $c$. Furthermore, for integers $n \geq 1$ and points $\underset{\tilde{C}}{x}, x^{\prime} \in S, \tilde{C}_{n}\left(x, x^{\prime}\right):=\sum_{r=1}^{n} \tilde{C}^{r}\left(x, x^{\prime}\right)$ is a continuous function. The sequence $\tilde{C}_{1}\left(x, x^{\prime}\right), \tilde{C}_{2}\left(x, x^{\prime}\right), \ldots$ is Cauchy since, for integers $p$ and $q, p>q \geq 1$,

$$
\left|\tilde{C}_{p}\left(x, x^{\prime}\right)-\tilde{C}_{q}\left(x, x^{\prime}\right)\right| \leq \sum_{r=q+1}^{p}\left|\tilde{C}^{r}\left(x, x^{\prime}\right)\right| \leq c \sum_{r=q+1}^{p}\|\tilde{C}\|^{r-2} \leq c \frac{\|\tilde{C}\|^{q-1}}{1-\|\tilde{C}\|}
$$

where the right-hand side is arbitrarily small for sufficiently large $q$ since $\|\tilde{C}\|<1$. Consequently,

$$
C\left(x, x^{\prime}\right):=\sum_{r=1}^{\infty} \tilde{C}^{r}\left(x, x^{\prime}\right), \quad x, x^{\prime} \in S
$$


is a well-defined continuous, symmetric function. This definition of $C$ is in accordance with Section 2.2, since it is easily seen that $C$ has eigenvalues $\lambda_{r}=\tilde{\lambda}_{r} /\left(1-\tilde{\lambda}_{r}\right)$ and eigenfunctions $e_{r}$. Observe also that $C$ is nonnegative, since each term $\tilde{C}^{r}$ is nonnegative. Moreover, as $\|\tilde{C}\|<1, D$ (see (9)) is well defined and satisfies

$$
D=-\sum_{r=0}^{\infty} \log \left(1-\tilde{\lambda}_{r}\right)=\sum_{n=1}^{\infty} \sum_{r=0}^{\infty} \frac{\tilde{\lambda}_{r}^{n}}{n}=\sum_{n=1}^{\infty} \frac{\operatorname{tr}\left(\tilde{C}^{n}\right)}{n},
$$

where the reversal of the order of summation requires the eigenvalues to be absolutely summable and less than 1 in absolute value. The latter sum may provide a fast way of approximating $D$.

By admitting negative eigenvalues of $C$ and noninteger values of $2 \alpha$, the connection with (2) is severed, so it may appear unlikely that the extensions of the permanental process to be established below are Cox processes in general. However, Clifford and Wei [4] considered a Cox process on $\mathbb{R}$ driven by a squared radial Ornstein-Uhlenbeck process, and their Equation (39) for the product densities $\rho^{(n)}$ can in fact be rewritten as a weighted permanent, showing that their Cox process is our extension of the permanental process for any value of $\alpha>0$ when $C$ is a stationary exponential covariance function. The issue at this point is connected with the infinite divisibility of squared Gaussian processes [10], [12]. Griffiths [12] proved that the square of a Gaussian Markov process is infinitely divisible, which explains why the Clifford-Wei process exists for all $\alpha>0$.

\subsection{Density of the extended process}

In [27, Theorem 1.4] it was shown that, for each $\alpha \geq 0$,

$$
f_{\alpha}(\boldsymbol{x}):=\mathrm{e}^{|S|-\alpha D} \operatorname{per}_{\alpha}[\tilde{C}](\boldsymbol{x})
$$

is the density of a point process in $S$ with cumulant generating function (5) and product density $\operatorname{per}_{\alpha}[C](\boldsymbol{x})$. Alternative and simpler proofs were provided in [21]; here we give a simpler proof of (13), since we shall refer to the same technique in the proof of Corollary 2. We let $\boldsymbol{X}_{\alpha}$ (or just $\boldsymbol{X}$ ) denote the permanental process with density (13). Note that $\boldsymbol{X}_{\alpha}=\varnothing$ almost surely if $\alpha=0$ or $\tilde{C} \equiv 0$.

Theorem 3. For each $\alpha \in \mathbb{R}_{+}$, the permanental process is well defined.

Proof. For integers $n \geq 1$,

$$
\begin{aligned}
\int_{S^{n}} \operatorname{cyp}[\tilde{C}](\boldsymbol{x}) \mathrm{d} \boldsymbol{x} & =\sum_{\{\sigma: \# \sigma=1\}} \sum_{r_{1}, \ldots, r_{n}=0}^{\infty} \int_{S^{n}} \prod_{j=1}^{n} \tilde{\lambda}_{r_{j}} e_{r_{j}}\left(x_{j}\right) e_{r_{j}}\left(x_{\sigma(j)}\right) \mathrm{d} \boldsymbol{x} \\
= & (n-1) ! \sum_{r_{1}, \ldots, r_{n}=0}^{\infty} \int_{S} \tilde{\lambda}_{r_{1}} e_{r_{1}}\left(x_{1}\right) e_{r_{n}}\left(x_{1}\right) \mathrm{d} x_{1} \\
& \times \int_{S} \tilde{\lambda}_{r_{2}} e_{r_{1}}\left(x_{2}\right) e_{r_{2}}\left(x_{2}\right) \mathrm{d} x_{2} \cdots \int_{S} \tilde{\lambda}_{r_{n}} e_{r_{n-1}}\left(x_{n}\right) e_{r_{n}}\left(x_{n}\right) \mathrm{d} x_{n} \\
= & (n-1) ! \sum_{r=0}^{\infty} \tilde{\lambda}_{r}^{n},
\end{aligned}
$$


where the second identity follows from Fubini's theorem, since each cyclic product has the same integral. Furthermore, with the convention that $\operatorname{cyp}[\tilde{C}](\varnothing)=0$,

$$
\sum_{n=0}^{\infty} \frac{1}{n !} \int_{S^{n}} \operatorname{cyp}[\tilde{C}](\boldsymbol{x}) \mathrm{d} \boldsymbol{x}=\sum_{n=1}^{\infty} \sum_{r=0}^{\infty} \frac{\tilde{\lambda}_{r}^{n}}{n}=D .
$$

For integers $r \geq 0$, define $\operatorname{cyp}^{(r)}[\tilde{C}](x)$ to be the sum of products over permutations having exactly $r$ cycles, with the convention that $\operatorname{cyp}^{(r)}[\tilde{C}](\varnothing)=1$ and $\operatorname{cyp}^{(r)}[\tilde{C}](x)=0$ if either $r>n(\boldsymbol{x})$ or $r=0<n(\boldsymbol{x})$. Then

$$
\begin{aligned}
\frac{D^{r}}{r !} & =\sum_{s_{1}=0}^{\infty} \cdots \sum_{s_{r}=0}^{\infty} \frac{1}{r ! s_{1} ! \cdots s_{r} !} \int_{S^{s_{1}}} \cdots \int_{S^{s_{r}}} \operatorname{cyp}[\tilde{\boldsymbol{C}}]\left(\boldsymbol{x}_{1}\right) \cdots \operatorname{cyp}[\tilde{\boldsymbol{C}}]\left(\boldsymbol{x}_{r}\right) \mathrm{d} \boldsymbol{x}_{1} \cdots \mathrm{d} \boldsymbol{x}_{r} \\
& =\sum_{n=0}^{\infty} \frac{1}{n !} \int_{S^{n}} \operatorname{cyp}^{(r)}[\tilde{C}](\boldsymbol{x}) \mathrm{d} \boldsymbol{x} .
\end{aligned}
$$

Thus, for any real $\alpha>0$, since $\operatorname{per}_{\alpha}[\tilde{\boldsymbol{C}}](\boldsymbol{x})=\sum_{r=0}^{\infty} \alpha^{r} \operatorname{cyp}^{(r)}[\tilde{\boldsymbol{C}}](\boldsymbol{x})$, we have

$$
\sum_{n=0}^{\infty} \frac{1}{n !} \int_{S^{n}} \operatorname{per}_{\alpha}[\tilde{C}](\boldsymbol{x}) \mathrm{d} \boldsymbol{x}=\mathrm{e}^{\alpha D} .
$$

This identity also holds for $\alpha=0$.

\subsection{Infinite divisibility}

The infinite divisibility of $f_{\alpha}$ means that if, for each integer $n \geq 1, \boldsymbol{Y}_{1}, \ldots, \boldsymbol{Y}_{n}$ are i.i.d. permanental processes with density $f_{\alpha / n}$, then the superposition $\bigcup_{j=1}^{n} \boldsymbol{Y}_{j}$ is a permanental process with density $f_{\alpha}$. By combining the convolution property (11) for weighted permanents with the density (13), we see that if $\boldsymbol{X}_{\alpha}$ and $\boldsymbol{X}_{\alpha^{\prime}}$ are independent permanental processes, then the superposition $\boldsymbol{X}_{\alpha} \cup \boldsymbol{X}_{\alpha^{\prime}}$ is a permanental process with density $f_{\alpha+\alpha^{\prime}}$. Consequently, since $f_{\alpha}$ exists for all $\alpha \in \mathbb{R}_{+}$, the permanental process is infinitely divisible. See also [27, Theorem 6.9].

In particular, this implies infinite divisibility of the number of points $N=n(\boldsymbol{X})$, with cumulant generating function

$$
\begin{aligned}
\log \mathrm{Ee}^{t N} & =-\alpha D+\log \left(\sum_{n=0}^{\infty} \frac{\mathrm{e}^{t n}}{n !} \int_{S^{n}} \operatorname{per}_{\alpha}[\tilde{C}](\boldsymbol{x}) \mathrm{d} x_{1} \cdots \mathrm{d} x_{n}\right) \\
& =-\alpha D+\log \left(\sum_{n=0}^{\infty} \frac{1}{n !} \int_{S^{n}} \operatorname{per}_{\alpha}\left[\mathrm{e}^{t} \tilde{C}\right](\boldsymbol{x}) \mathrm{d} x_{1} \cdots \mathrm{d} x_{n}\right) \\
& =-\alpha D-\alpha \sum_{r=0}^{\infty} \log \left(1-\mathrm{e}^{t} \tilde{\lambda}_{r}\right), \quad t \leq-\log \|\tilde{C}\|
\end{aligned}
$$

Thus, $N$ is distributed in the same way as the sum of independent negative binomial random variables with probability density functions

$$
\frac{\Gamma(n+\alpha)}{\Gamma(\alpha) n !} \tilde{\lambda}_{r}^{n}\left(1-\tilde{\lambda}_{r}\right)^{\alpha}, \quad n=0,1, \ldots,
$$


for $r=0,1, \ldots$ (taking $0^{0}=1$ ). Consequently, $N$ is overdispersed and

$$
\mathrm{E} N=\alpha \sum_{r=0}^{\infty} \frac{\tilde{\lambda}_{r}}{1-\tilde{\lambda}_{r}}=\alpha \sum_{r=0}^{\infty} \lambda_{r}, \quad \operatorname{var} N=\alpha \sum_{r=0}^{\infty} \frac{\tilde{\lambda}_{r}}{\left(1-\tilde{\lambda}_{r}\right)^{2}}=\alpha \sum_{r=0}^{\infty} \lambda_{r}\left(1+\lambda_{r}\right) \text {. }
$$

The probability that $\boldsymbol{X}_{\alpha}$ is empty is $\mathrm{e}^{-\alpha D}$, which tends to 1 as $\alpha$ tends to 0 . The conditional distributions given $\boldsymbol{X}_{\alpha} \neq \varnothing$ have a nontrivial limit density $f_{0}(\boldsymbol{x} \mid$ not $\varnothing)$ as $\alpha \rightarrow 0$, and we let $W$ denote the number of points in this limiting process.

Corollary 1. For finite, nonempty point configurations $\boldsymbol{x} \subset S$,

$$
f_{0}(\boldsymbol{x} \mid \text { not } \varnothing)=\mathrm{e}^{|S|} \operatorname{cyp}[\tilde{\boldsymbol{C}}](\boldsymbol{x}) / D
$$

and

$$
\mathrm{P}(W=n)=D^{-1} \sum_{r=0}^{\infty} \frac{\tilde{\lambda}_{r}^{n}}{n}, \quad n=1,2, \ldots
$$

Moreover, $\boldsymbol{X}_{\alpha}$ is a Poisson randomization; that is, if $R$ is a Poisson distributed random variable with mean $\alpha D$, then $\boldsymbol{X}_{\alpha}$ is distributed in the same way as the superposition of $R$ i.i.d. point processes with density $f_{0}(\boldsymbol{x} \mid$ not $\varnothing)$.

Proof. The conditional density given that $\boldsymbol{X}_{\alpha}$ is nonempty is

$$
\frac{\mathrm{e}^{|S|} \operatorname{per}_{\alpha}[\tilde{\boldsymbol{C}}](\boldsymbol{x})}{1-\mathrm{e}^{-\alpha D}},
$$

which tends to (17) as $\alpha$ tends to 0 . Equation (15) shows that $f_{0}(\boldsymbol{x} \mid$ not $\varnothing$ ) is a density with respect to the unit-rate Poisson process on $S$. Combining (14) and (17) yields (18). Finally, by the same arguments as in the proof of Theorem 3, for any event $F$ of finite point configurations $\boldsymbol{x} \subset S$,

$$
\begin{aligned}
\mathrm{P}\left(\boldsymbol{X}_{\alpha} \in F\right)= & \sum_{n=0}^{\infty} \frac{\mathrm{e}^{-\alpha D}}{n !} \int_{S^{n}} \mathbf{1}_{\{\boldsymbol{x} \in F\}} \operatorname{per}_{\alpha}[\tilde{C}](\boldsymbol{x}) \mathrm{d} \boldsymbol{x} \\
= & \sum_{r=0}^{\infty} \frac{(\alpha D)^{r} \mathrm{e}^{-\alpha D}}{r !} \sum_{s_{1}=0}^{\infty} \cdots \sum_{s_{r}=0}^{\infty} \frac{\mathrm{e}^{-|S|}}{s_{1} !} \cdots \frac{\mathrm{e}^{-|S|}}{s_{r} !} \\
& \times \int_{S^{s_{1}}} \cdots \int_{S^{s_{r}}} \mathbf{1}_{\left\{\boldsymbol{x}_{1} \cup \cdots \cup \boldsymbol{x}_{r} \in F\right\}} f_{0}\left(\boldsymbol{x}_{1} \mid \operatorname{not} \varnothing\right) \cdots f_{0}\left(\boldsymbol{x}_{r} \mid \operatorname{not} \varnothing\right) \mathrm{d} \boldsymbol{x}_{1} \cdots \mathrm{d} \boldsymbol{x}_{r},
\end{aligned}
$$

from which it follows that $\boldsymbol{X}_{\alpha}$ is a Poisson randomization.

From (18) we obtain the expected number of points:

$$
\mathrm{E} W=D^{-1} \sum_{r=0}^{\infty} \frac{\tilde{\lambda}_{r}}{1-\tilde{\lambda}_{r}}=\sum_{r=0}^{\infty} \frac{\lambda_{r}}{D} .
$$

As an example of such a limit process, let $S=[0,2 \pi]$ and

$$
\tilde{C}\left(x, x^{\prime}\right)=\frac{\theta\left(1-\cos \left(x-x^{\prime}\right)\right)}{2 \pi}, \quad 0<\theta<1,
$$

so $\tilde{C}(x, x) \geq 0$. The nonzero eigenvalues of $\tilde{C}$ are $\theta$ and $-\theta / 2$ and have multiplicities one and two, respectively. Note that $\tilde{C}$ is not positive semidefinite and that $\mathrm{E} W$ is an increasing function of $\theta$ with range $(0, \infty)$. 


\subsection{Simulation}

A random point configuration with density $f_{0}(\cdot \mid$ not $\varnothing)$ is obtained by first generating the size $W=n$, followed by a list of $n$ points in $S$ with joint density proportional to

$$
\tilde{C}\left(x_{1}, x_{2}\right) \tilde{C}\left(x_{2}, x_{3}\right) \cdots \tilde{C}\left(x_{n}, x_{1}\right),
$$

and then ignoring the order. The joint density of the ordered values is not invariant under permutation, but it is of Gibbs type with a cyclic graph in which each component has two neighbours. Gibbs sampling or another Metropolis-Hastings algorithm may be used to simulate from the 'full conditionals' with densities

$$
\pi\left(x_{i} \mid \cdots\right) \propto \tilde{C}\left(x_{i-1}, x_{i}\right) \tilde{C}\left(x_{i}, x_{i+1}\right),
$$

where $x_{n+1}=x_{1}$.

This, together with the Poisson randomization in Corollary 1, provides one way of simulating from $f_{\alpha}$. If $C$ is positive semidefinite and $2 \alpha$ is a positive integer, a faster algorithm exploits the doubly stochastic construction of the Cox process $\boldsymbol{X}_{\alpha}$, in that we simulate first the Gaussian processes and second the Poisson process $\boldsymbol{X}_{\alpha} \mid \boldsymbol{\Lambda}$. In the special case of a stationary exponential covariance function $C\left(x, x^{\prime}\right)=\beta \exp \left(-\delta\left|x-x^{\prime}\right|\right), x, x^{\prime} \in \mathbb{R}$, where $\beta$ and $\delta$ are positive parameters, Clifford and Wei [4] established an equivalence between our permanental process and a simple stationary immigration-birth-death process, which is easy to simulate.

\section{Two temporal extensions}

Spatial birth-death processes satisfying a detailed-balance condition with respect to $f_{\alpha}$ can easily be constructed when a birth is the addition of a single point and a death is the deletion of a single point [26]. The detailed-balance condition requires the evaluation of the so-called Papangelou conditional intensity $f(\boldsymbol{x} \cup\{x\}) / f(\boldsymbol{x})$, which is a ratio of permanents, namely $\operatorname{per}_{\alpha}[\tilde{C}](\boldsymbol{x} \cup\{x\}) / \operatorname{per}_{\alpha}[\tilde{C}](\boldsymbol{x})$. Below we consider two other spatio-temporal constructions.

\subsection{An accretion process with independent increments}

The Poisson randomization established in Corollary 2 implies that there exists a coupling construction of the permanental processes $\boldsymbol{X}_{\alpha}$ for all $\alpha \in \mathbb{R}_{+}$. By interpreting $\alpha=t$ as time, we obtain a continuous-time jump process $\left(\boldsymbol{X}_{t}\right)_{t \geq 0}$ where we have 'evolution by accretion' and 'i.i.d. increments'.

The process is constructed as follows: $\left(\boldsymbol{X}_{t}\right)_{t \geq 0}$ is constant almost everywhere except at the jump times, which are independent of the jumps; the jump times constitute a homogeneous Poisson process on $\mathbb{R}_{+}$with rate $D$; the jumps are i.i.d. point processes with density $f_{0}(\boldsymbol{x} \mid$ not $\varnothing)$; and $\boldsymbol{X}_{t}$ is the superposition of the jumps happening before or at time $t$. Note that $\boldsymbol{X}_{0}=\varnothing$.

By Corollary $2, \boldsymbol{X}_{t}$ is a permanental process with density $f_{t}$. The jump process is clearly Markovian and increasing $\left(\boldsymbol{X}_{s} \subseteq \boldsymbol{X}_{t}\right.$ if $\left.0 \leq s<t\right)$. Hence, for each $s, 0 \leq s \leq s+t$, the increments $\boldsymbol{X}_{s}$ and $\boldsymbol{X}_{s+t} \backslash \boldsymbol{X}_{s}$ are independent and have respective densities $f_{s}$ and $f_{t}$.

\subsection{Second temporal extension}

In this section we assume that $2 \alpha$ is a positive integer and that the conditions of Section 2.2 are satisfied.

Consider a spatio-temporal Cox process for which the conditional intensity function at $(x, t)$ is $\Lambda(x)$, from (2), which is constant in time. Let $t>0$ be fixed and let $X \subset S$ be the set of points occurring in $[0, t]$. That is to say, $\boldsymbol{X}$ records the position of each point, but not the 
time of occurrence or the sequential order. Given $\Lambda$, the process is Poisson with intensity function $t \Lambda(x)$ for $x \in S$. According to Theorem 2, the density of $\boldsymbol{X}$ is given by (10) with $\lambda_{r}$ replaced by $t \lambda_{r}$. Thus, $\tilde{\lambda}_{r}=t \lambda_{r} /\left(1+t \lambda_{r}\right)$, and the corresponding covariance function is denoted by $\tilde{C}_{t}$. Given that $n(\boldsymbol{X})=n$, the conditional density of the points in $S^{n}$ is proportional to $\operatorname{per}_{\alpha}\left[\tilde{C}_{t}\right]\left(x_{1}, \ldots, x_{n}\right)$.

For inverse sampling, the number of points is fixed and the process is observed until the time, $T_{n}$, at which $n \geq 1$ points have occurred. What then is the joint density of $T_{n}$ and the $n$ points? Let $\Gamma=\int_{S} \Lambda(x) \mathrm{d} x$. Given $\Lambda$, the points are i.i.d. in $S$ with density $\Lambda(x) / \Gamma$ and $T_{n}$ has the gamma distribution with shape parameter $n$ and mean $n / \Gamma$, independent of the $n$ points. The conditional joint density at $\left(x_{1}, \ldots, x_{n}, t\right)$ is thus

$$
\frac{\Lambda\left(x_{1}\right) \cdots \Lambda\left(x_{n}\right)}{\Gamma^{n}} \frac{t^{n-1} \Gamma^{n} \mathrm{e}^{-t \Gamma}}{(n-1) !} .
$$

From the proof of Theorem 2, the unconditional joint density is

$$
\frac{t^{-1} \operatorname{per}_{\alpha}\left[\tilde{C}_{t}\right]\left(x_{1}, \ldots, x_{n}\right)}{(n-1) ! \prod_{0}^{\infty}\left(1+t \lambda_{r}\right)^{\alpha}}
$$

and the marginal density on $S^{n}$ of the points is

$$
f_{n}\left(x_{1}, \ldots, x_{n}\right)=\int_{0}^{\infty} \frac{t^{-1} \operatorname{per}_{\alpha}\left[\tilde{C}_{t}\right]\left(x_{1}, \ldots, x_{n}\right)}{(n-1) ! \prod_{0}^{\infty}\left(1+t \lambda_{r}\right)^{\alpha}} \mathrm{d} t .
$$

As will be shown in Section 5, unless $C$ is proportional to a projection this is different from the conditional density obtained in the preceding paragraph.

The eigenvalues $\tilde{\lambda}_{r}=t \lambda_{r} /\left(1+t \lambda_{r}\right)$ of $\tilde{C}_{t}$ are strictly less than 1 , but increase in $t$ with limit 1 if $\lambda_{r}>0$ and limit 0 otherwise, as $t \rightarrow \infty$. Thus, if $C$ has finite rank then the limit $\lim _{t \rightarrow \infty} \tilde{C}_{t}$ is the orthogonal projection having the same range as $C$. Moreover, if, for example, $\lambda_{r}=\mathrm{e}^{-r}$ and $\delta>0$, then the eigenvalues of $\tilde{C}_{t}$ are near 1 for $r<\log t-\delta \log \log t$ and near 0 for $r>\log t+\delta \log \log t$. We interpret this result as stating that $\tilde{C}_{t}$ is approximately a projection of rank $\log t$ when $t$ is large. These special permanental processes are studied in the next section.

\section{The special permanental process}

Let $Q$ be a projection of rank $m$, i.e.

$$
\int_{S} Q\left(x_{1}, x\right) Q\left(x, x_{2}\right) \mathrm{d} x=Q\left(x_{1}, x_{2}\right)
$$

and $\int_{S} Q(x, x) \mathrm{d} x=m$. This means that $Q$ has $m$ unit eigenvalues and the others are 0 . It is assumed throughout this section that $\kappa>0$ is a parameter and that the covariance function $C=$ $\kappa Q$ is a positive multiple of the projection; equivalently, $\tilde{C}=(\kappa /(1+\kappa)) Q$. The associated point process $\boldsymbol{X}_{\alpha}$ is deemed special on account of its striking and unusual properties.

Corollary 2. Suppose that $\alpha>0$. For any finite point configuration $\boldsymbol{x} \subset S$, the special permanental process $\boldsymbol{X}$ has density

$$
f(\boldsymbol{x})=\mathrm{e}^{|S|}(1+\kappa)^{-n(\boldsymbol{x})-\alpha m} \operatorname{per}_{\alpha}[C](\boldsymbol{x})
$$


and the number $N$ of points in $\boldsymbol{X}$ follows a negative binomial distribution with density

$$
p_{n}=\frac{\Gamma(n+m \alpha)}{\Gamma(m \alpha) n !}\left(\frac{\kappa}{1+\kappa}\right)^{n}\left(\frac{1}{1+\kappa}\right)^{m \alpha}, \quad n=0,1, \ldots
$$

Furthermore, conditional on $N=n$, the joint density of the $n$ points in $\boldsymbol{X}$ is

$$
f_{n}\left(x_{1}, \ldots, x_{n}\right)=\operatorname{per}_{\alpha}[Q]\left(x_{1}, \ldots, x_{n}\right) \frac{\Gamma(m \alpha)}{\Gamma(n+m \alpha)},
$$

and

$$
\int_{S} f_{n+1}\left(x_{1}, \ldots, x_{n}, x\right) \mathrm{d} x=f_{n}\left(x_{1}, \ldots, x_{n}\right) .
$$

Proof. We have $\operatorname{per}_{\alpha}[\tilde{C}](\boldsymbol{x})=\operatorname{per}_{\alpha}[C](\boldsymbol{x}) /(1+\kappa)^{n(\boldsymbol{x})}$. Since $\tilde{\lambda}_{r}=\kappa /(1+\kappa)$ for $m$ eigenvalues and $\tilde{\lambda}_{r}=0$ for the others, $\prod_{0}^{\infty}\left(1+\lambda_{r}\right)=(1+\kappa)^{m}$. Hence, (21) follows immediately from Theorem 3. By (16), $N$ has cumulant generating function $-m \alpha \log \left(1+\kappa\left(1-\mathrm{e}^{t}\right)\right)$, from which (22) follows. Furthermore, (23) follows from (21) and (22) and the usual relation between $f$ and $p_{n} f_{n}$ :

$$
f(\boldsymbol{x})=p_{n} f_{n}\left(x_{1}, \ldots, x_{n}\right) \frac{\mathrm{e}^{|S|}}{n !} .
$$

Finally, (24) follows straightforwardly from (20) and (23).

Equation (24) is Kolmogorov's consistency condition for a stochastic process with marginal densities $f_{n}$. In other words, to each projection $Q$ there corresponds an infinitely exchangeable process taking values in $S$ for which the $n$-dimensional joint density is $f_{n}$. Furthermore, (21) implies that $\left(n(\boldsymbol{x}), \rho^{(n(\boldsymbol{x}))}(\boldsymbol{x})\right)$ is a minimal sufficient statistic, and (23) states that $f_{n}$ is proportional to $\rho^{(n)}$. In general, for other nontrivial Cox processes such as log Gaussian or shot noise processes, no simple relationship exists connecting product densities with the density of the process.

Again consider the space-time setting of Section 4.2, where now $C=\kappa Q$. Suppose that the point configuration $\boldsymbol{x}=\left\{x_{1}, \ldots, x_{n}\right\}$ has been observed by inverse sampling with fixed $n$, and that we wish to predict where the next point, $X_{n+1}$, is likely to occur. Since the density $f_{n}$ in (19) reduces to that in (23), and since $f_{n}$ is the marginal density of $f_{n+1}$, the conditional density of $X_{n+1}$ at $x$ is

$$
\frac{f_{n+1}\left(x_{1}, \ldots, x_{n}, x\right)}{f_{n}\left(x_{1}, \ldots, x_{n}\right)}=\frac{\operatorname{per}_{\alpha}[Q]\left(x_{1}, \ldots, x_{n}, x\right)}{(m \alpha+n) \operatorname{per}_{\alpha}[Q]\left(x_{1}, \ldots, x_{n}\right)} .
$$

This predictive density is in fact the Bayes estimate of the intensity function $\Lambda(x) / \Gamma$, i.e. the conditional expected value of the normalized intensity function at $x$ given the observed point configuration $\boldsymbol{x}$.

\section{The determinantal process}

An analogous theory, in which the fermion process replaces the boson process, follows similar lines, extending the work of [7] in a different direction. We sketch this below.

Suppose that $C$ satisfies the conditions of Section 2.2, i.e. $C$ is a covariance function with spectral representation (6) such that $\sum_{0}^{\infty} \lambda_{r}<\infty$. The fermion (or electron) process is a finite point process with density

$$
\tilde{f}_{1}(\boldsymbol{x}):=\mathrm{e}^{|S|-D} \operatorname{det}[C](\boldsymbol{x})
$$


with respect to the unit-rate Poisson process on $S$, and its $n$ th-order product density, $\tilde{\rho}^{(n)}$, is given by

$$
\tilde{\rho}^{(n)}(\boldsymbol{x}):=\operatorname{det}[\tilde{\boldsymbol{C}}](\boldsymbol{x})
$$

(see [2], [6], and [18]). Note that $\operatorname{det}[C](\boldsymbol{x})$ and $\operatorname{det}[\tilde{\boldsymbol{C}}](\boldsymbol{x})$ can be negative if $C$ is not positive semidefinite.

The determinant polynomial

$$
\operatorname{det}_{\alpha}[C]\left(x_{1}, \ldots, x_{n}\right):=\operatorname{per}_{-\alpha}[-C]\left(x_{1}, \ldots, x_{n}\right)
$$

with $\alpha^{\# \sigma} \operatorname{sgn}(\sigma)=(-1)^{n}(-\alpha)^{\# \sigma}$ in place of $\alpha^{\# \sigma}$ also satisfies the convolution property (11). Consequently, for positive integer $\alpha$, the family of point processes with density

$$
\tilde{f}_{\alpha}(\boldsymbol{x}):=\mathrm{e}^{|S|-\alpha D} \operatorname{det}_{\alpha}[C](\boldsymbol{x})
$$

is closed under independent superposition (see also [27, Lemma 3.3]). A point process with density $\tilde{f}_{\alpha}$ is called a determinantal process. In contrast to the permanental process, and unless the process is Poisson (i.e. $C\left(x, x^{\prime}\right)=0$ for $\left.x \neq x^{\prime}\right)$, the points of the determinantal process tend to repel one another.

Most of the results established for permanental processes have a dual form for determinantal processes with $C$ and $\tilde{C}$ interchanged. For example, the $n$ th-order product density is

$$
\tilde{\rho}^{(n)}(\boldsymbol{x}):=\operatorname{det}_{\alpha}[\tilde{C}](\boldsymbol{x})
$$

(see also [27, Theorem 4.1]). Furthermore, the special determinantal process in which $C=\kappa Q$ is proportional to a projection of rank $m$ has conditional densities

$$
\operatorname{det}_{\alpha}[Q]\left(x_{1}, \ldots, x_{n}\right) \frac{\Gamma(m \alpha-n+1)}{\Gamma(m \alpha+1)}
$$

for $n \leq m \alpha$ only, and these satisfy the Kolmogorov consistency condition up to this order. Furthermore, the number of points is binomial with index $m \alpha$ and parameter $\kappa /(1+\kappa)$, making it clear that the special determinantal process is defined for integer $\alpha$ only.

In general, the determinantal process cannot be extended to $\alpha \in(0,1)$ : if we claim $f_{\alpha}$ to be a density then, since $\tilde{\rho}^{(2)}\left(x, x^{\prime}\right)=\alpha^{2}\left(\tilde{C}(x, x) \tilde{C}\left(x^{\prime}, x^{\prime}\right)-\tilde{C}\left(x, x^{\prime}\right)^{2} / \alpha\right)$ has to be nonnegative, continuity of the covariance function $\tilde{C}$, for example, implies that $\alpha \geq 1$.

\section{Acknowledgements}

We are grateful to a referee for drawing our attention to [27]. Comments by Alexander Barvinok, Horia Cornean, Arne Jensen, Bob Griffiths, Mark Jerrum, Joseph Mecke, Gareth Roberts, Alistar Sinclair, and Eric Vigoda are also acknowledged. JM thanks the Department of Statistics, University of Chicago, for their kind hospitality. This research was funded by the Danish Natural Science Research Council and the NSF, grant no. DMS-0305009.

\section{References}

[1] Barvinok, A. I. (1996). Two algorithmic results for the traveling salesman problem. Math. Operat. Res. 21, 65-84.

[2] Benard, C. AND Macchi, O. (1973). Detection and emission processes of quantum particles in a chaotic state. J. Math. Phys. 14, 155-167. 
[3] Brix, A. (1999). Generalized gamma measures and shot-noise Cox processes. Adv. Appl. Prob. 31, 929-953.

[4] Clifford, P. And Wei, G. (1993). The equivalence of the Cox process with squared radial Ornstein-Uhlenbeck intensity and the death process in a simple population model. Ann. Appl. Prob. 3, 863-873.

[5] Cox, D. R. And Isham, V. (1980). Point Processes. Chapman and Hall, London.

[6] Daley, D. J. And Vere-Jones, D. (2003). An Introduction to the Theory of Point Processes, Vol. I, Elementary Theory and Methods, 2nd edn. Springer, New York.

[7] Diaconis, P. And Evans, S. (2000). Immanants and finite point processes. J. Combinatorial Theory 91, 305-321.

[8] Dieudonné, J. (1969). Foundations of Modern Analysis. Academic Press, New York.

[9] Diggle, P. J. (2003). Statistical Analysis of Spatial Point Patterns, 2nd edn. Arnold, London.

[10] Eisenbaum, N. (2003). On the infinite divisibility of squared Gaussian processes. Prob. Theory Relat. Fields 125, 381-392.

[11] Grandell, J. (1976). Doubly Stochastic Poisson Processes (Lecture Notes Math. 529). Springer, Berlin.

[12] Griffiths, R. C. (1984). Characterization of infinitely divisible multivariate gamma distributions. J. Multivariate Anal. 15, 13-20.

[13] Griffiths, R. C. ANd Milne, R. K. (1987). A class of infinitely divisible multivariate negative binomial distributions. J. Multivariate Anal. 22, 13-23.

[14] Hough, J. B., Krishnapur, M., Peres, Y. and Virág, B. (2006). Determinantal processes and independence. Prob. Surveys 3, 206-229.

[15] IsSERLIS, L. (1918). On a formula for the product-moment coefficient of any order of a normal frequency distribution in any number of variables. Biometrika 12, 134-139.

[16] Jerrum, M. R., Sinclair, A. and Vigoda, E. (2004). A polynomial-time approximation algorithm for the permanent of a matrix with non-negative entries. J. Assoc. Comput. Mach. 51, 671-697.

[17] MaCchi, O. (1971). Distribution statistique des instants d'émission des photoélectrons d'une lumière thermique. C. R. Acad. Sci. Paris A 272, 437-440.

[18] Macchi, O. (1975). The coincidence approach to stochastic point processes. Adv. Appl. Prob. 7, 83-122.

[19] Malyshev, V. (1980). Cluster expansions in lattice models of statistical physics and the quantum theory of fields. Russian Math. Surveys 35, 1-62.

[20] McCullagh, P. (1984). Tensor notation and cumulants of polynomials. Biometrika 71, 461-476.

[21] McCullagh, P. and Møller, J. (2005). The permanent process. Tech. Rep. R-2005-29, Department of Mathematical Sciences, Aalborg University.

[22] Minc, H. (1978). Permanents (Encyclopaedia Math. Appl. 6). Addison-Wesley, Reading, MA.

[23] Møller, J. (2003). Shot noise Cox processes. Adv. Appl. Prob. 35, 4-26.

[24] Møller, J. And Waagepetersen, R. P. (2003). Statistical Inference and Simulation for Spatial Point Processes. Chapman and Hall/CRC, Boca Raton, FL.

[25] Møller, J., Syversveen, A. R. and Waagepetersen, R. P. (1998). Log Gaussian Cox processes. Scand. J. Statist. 25, 451-482.

[26] RiPLey, B. D. (1977). Modelling spatial patterns (with discussion). J. R. Statist. Soc. B 39, 172-212.

[27] Shirai, T. and TaKahashi, Y. (2003). Random point fields associated with certain Fredholm determinants. I. Fermion, Poisson and boson point processes. J. Funct. Anal. 205, 414-463.

[28] Soshnikov, A. (2000). Determinantal random point fields. Russian Math. Surveys 55, 923-975.

[29] Stoyan, D., Kendall, W. S. And Mecke, J. (1995). Stochastic Geometry and Its Applications, 2nd edn. John Wiley, Chichester.

[30] Valiant, L. G. (1979). The complexity of computing the permanent. Theoret. Comput. Sci. 8, 189-201.

[31] Van Lieshout, M. N. M. (2000). Markov Point Processes and Their Applications. Imperial College Press, London.

[32] Vere-Jones, D. (1988). A generalization of permanents and determinants. Linear Algebra Appl. 111, 119-124. 